\title{
Fehlermanagement im Spital - ein Beispiel aus unserer Frauenklinik
}

\author{
D. Haenggi, S. Heinzl
}

Ausländische Studien haben ergeben, dass Hunderte von Patientinnen und Patienten jährlich durch vermeidbare medizinische Fehler geschädigt oder gar getötet werden. Entsprechende Hochrechnungen für die Schweiz haben für grosses Echo in den Medien gesorgt. Bislang gibt es weder in der Schweiz noch in Europa eine umfassende Statistik über medizinische Behandlungsfehler. Studien aus den USA zeigen, dass jährlich etwa 120000 Patienten an Behandlungsfehlern sterben. Der ärztliche Kunstfehler wird als fünftwichtigste Todesursache in den Vereinigten Staaten angesehen [1]. Fehlervorwürfe betreffen vor allem die operativen Disziplinen und hierbei im besonderen die Spitalärzte. Allerdings ist davon auszugehen, dass dies kein getreues Abbild der Realität ist, sondern eher ein Wahrnehmungseffekt. Denn sieht man von fachspezifischen Problemen $a b$, werden vorrangig folgende drei Gründe für die Entstehung von medizinischen Behandlungsfehlern deutlich: organisatorische Defizite mit Kommunikationsmängeln, Dokumentationsdefizite und die Behandlung von Patientinnen und Patienten in nicht optimal geeigneten Einrichtungen [2].

Präventionsansätze beinhalten die Einführung von Qualitätssicherungsund Managementinstrumenten in Krankenhäusern sowie ärztlichen Praxen. Dazu kommen themenbezogene Analysen zusammengefasster Fehlermeldungen sowie die Entwicklung von Leitlinien. Wünschenswert wäre ein nationales Projekt zur Analyse von ausreichend grossen Zahlen vermuteter Behandlungsfehler mit Ableitung entsprechender Vermeidungsstrategien. Nur so kann aus dem ursprünglich geschützten Privatbereich einer Klinik eine Aufgabe von hohem gesamtgesellschaftlichem Interesse entstehen.

\section{Einleitung}

Das Institute of Medicine rechnete 1999 im Bericht «To Err is Human» mit jährlich 90000 iatrogenen Todesfällen in US-Spitälern [3]. Die Anzahl der Prozesse wegen angeblicher Behandlungsfehler ist in den letzten Jahren dramatisch gestiegen. Ein Beispiel: Im Oktober 2002 musste eine Schwangerschaft wegen Implantation von fremden Embryonen in England abgebrochen werden, da die Röhrchen, die die befruchteten Embryonen enthielten, falsch beschriftet waren. Die IVF-Klinik wurde daraufhin geschlossen.

Gerade auch in der Geburtshilfe sind deutliche Steigerungen anzutreffen. Die Haftpflichtschäden in den Krankenhäusern haben sich in den letzten 10 Jahren mehr als verdoppelt. In Deutschland geht man von etwa 35000 Haftpflichtschadensfällen pro Jahr aus, mit einer $\mathrm{Zu}$ nahme von $75 \%$ innert 5 Jahren [4]. Allerdings besteht auch hier ein deutlicher Mangel an aussagefähigen epidemiologischen Untersuchungen und die ausländischen Daten sind nur ein- geschränkt zu verwenden. Interessant ist auch die Tatsache, dass die Fachdiskussion um Identifizierung und Vermeidung von Fehlern in der Medizin im internationalen Schrifttum mit Zurückhaltung geführt wird. Dies ist um so erstaunlicher, als sich die Laienpresse seit langem umfassend dieser Problematik widmet [5].

Ebenso rasant entwickelten sich die Schadenssummen. So werden heute für einen Schaden in der Geburtshilfe bis zu 4 Mio. Euro bezahlt, vor 10 Jahren waren es noch 300000 Euro [6]. Die damit verbundenen Kosten sind enorm. Die Schlichtungsstelle für Arzthaftpflichtfragen der Norddeutschen Ärztekammer berichtet über eine Verdoppelung der begründeten Schadenersatzansprüche innerhalb der letzten 10 Jahre [7]. Der durchschnittliche Schadensaufwand pro Fall liegt bei der DBV Winterthur (108000 versicherte Ärzte) bei 25000 Euro. Er steigt bei Schäden in Gynäkologie und Geburtshilfe auf über 100000 Euro pro Fall an [8]. Gemäss Ollenschläger sind in Deutschland die Schadensaufwendungen in den letzten 5 Jahren um 100\% gestiegen [9].

Rall rechnete die Zahlen der USA auf Deutschland hoch und kam umgerechnet pro Jahr auf 16000-40 000 Tote aufgrund medizinischer Behandlungsfehler in Deutschland. Damit wären medizinische Fehler auf Platz 8 aller Todesursachen [10]. Vincent et al. rechneten für das ganze britische Gesundheitswesen 1 Mia. Pfund pro Jahr [11]. Er berichtete über ähnliche Zahlen wie das Institute of Medicine aus England und zeigte, dass bei vielen Patienten unerwünschte Ereignisse auftreten [11]. Daraus wurden Zahlen für die Schweiz hochgerechnet, welche zum Teil heftiger Kritik ausgesetzt waren. Der Gesamtumfang des volkswirtschaftlichen Schadens lässt sich daraus allerdings nicht kalkulieren, er dürfte aber beträchtlich sein. Gemäss Scheidegger sind hingegen der Wechsel von einer «culture of blame» zu einer «culture of safety» wichtiger als exakte Zahlen [12]. Denn nur so sei es überhaupt möglich, das Problemausmass zu identifizieren.

Dementsprechend sehen sich die heutigen Kliniken mit wachsenden Patientenansprüchen konfrontiert. Das Spitalimage steht auf dem Spiel. Neustrukturierungen und Einsparungsmassnah- 
men müssen bei steigender Qualität vorgenommen werden. Aus diesem Grund wird auch in $\mathrm{Zu}-$ kunft ein erhöhter Bedarf an neuen Berufsgattungen und Spezialisten dazu beitragen, Kosten zu reduzieren und eine hohe Qualität sicherzustellen. So z. B. das Krankenhaus-Risk-Management, welches sich aus Experten aus den Bereichen Medizin, Pflege und Qualitätsmanagement zusammensetzt und interdisziplinär mit Juristen, Versicherern und Ärzten verschiedener Fachrichtungen zusammenarbeitet. Dass die Medizin eine Hochrisikobranche ist, welche sich bis anhin im Umgang mit Fehlern sehr schwertat, ist uns allen bekannt. Auch die anhand von internationalen Studien hochgerechneten Zahlen für die Schweiz mit einigen Tausend Menschen, welche jährlich in Akutkliniken wegen vermeidbarer Fehler sterben, sind uns in der Öffentlichkeit zur Genüge vorgeführt worden. Es ist somit höchste Zeit, dass dem Risk Management in den Spitälern höchste Priorität eingeräumt wird [13].

Um nun das Ausmass des Problems überhaupt zu erkennen und erste Massnahmen ableiten zu können, werden an verschiedenen Institutionen Fehlermanagementsysteme eingerichtet. Viele Fehler wären vermeidbar. Warum ist es aber gerade für uns, in Medizinalberufen Tätige so schwer, mit Fehlern umzugehen? Das Problem ist, dass wir wissen, dass wir uns keine Fehler leisten dürfen und Verantwortung für unsere Patienten übernehmen müssen, was uns zu ständiger Perfektion im täglichen Ablauf zwingt. Solche Systeme, deren Zuverlässigkeit auf dem perfekten Funktionieren des einzelnen basieren, müssen aber früher oder später versagen, ganz einfach, weil niemand fähig ist, immer perfekte Arbeit zu leisten. Menschliche Fehlleistungen sind normale tägliche Erscheinungen, welche insbesondere bei Routinehandlungen vermehrt vorkommen. Ein weiteres Problem in Spitälern ist die starke Hierarchie, wobei versucht wird, in allen Fehlern einen Schuldigen zu finden und ihn entsprechend $\mathrm{zu}$ bestrafen. Die logische Folge davon ist, dass über Fehler, die sich vertuschen lassen, nicht berichtet oder diskutiert wird. Nach Kehrer [14] wiegen wir uns deshalb in einer falschen Sicherheit. Untersuchungen haben gezeigt, dass sich etwa 50mal mehr Zwischenfälle ereignen, als spitalintern bekannt werden. Deshalb ist es wichtig, dass alle Mitarbeiterinnen und Mitarbeiter wissen, dass ihre Meldung zu einer konstruktiven, objektiven Abklärung der Ereignisse führt, die allein zum Ziel hat, eine Wiederholung zu verhindern. Es geht also nicht darum, Sanktionen zu ergreifen, sondern eine Atmosphäre des gegenseitigen Vertrauens zu schaffen, die Kommunikation zu verbessern und Bewältigungsstrategien zu entwickeln, damit die Zwischenfälle nicht mehr in diesem Ausmass geschehen können.

Vor dem Hintergrund der international zunehmenden Diskussion sammeln wir an unserer Frauenklinik seit drei Jahren Erfahrungen im Umgang mit Fehlern. Dabei etablierten wir ein «critical incidents reporting system» mit dem Ziel, Erfahrungen in diesem Bereich zu sammeln und das Ausmass des Problems zu erkennen. Es gibt in einem Klinikalltag eine ganze Menge an Unzulänglichkeiten, über die man sich täglich ärgert. Wo Menschen arbeiten werden Fehler gemacht, auch in der Medizin. Niemand wird dies bestreiten. Einige Fehler weisen jedoch Gesetzmässigkeiten auf, die auf strukturellen, apparativen, organisatorischen oder auch personellen Vorgaben beruhen.

Die Einführung eines Qualitätssystems bietet Anlass, sich mit diesem Thema ernsthaft auseinanderzusetzen. Nur durch die systematische Erfassung von Fehlern kann beurteilt werden, welche aufgrund ihrer Häufigkeit oder Kostenintensität schwerwiegende Auswirkungen zeigen [15]. Die Analyse der häufigen, meist «irrelevanten» Ereignisse an der Basis des Eisbergs ist für die langfristige Reduktion der schweren Zwischenfälle an der Spitze sehr wichtig.

Dass jeder erkannte Irrtum oder Missstand und die daraus hoffentlich folgende Schadensverhinderung ein Gewinn ist, von diesem Denkprinzip sind wir noch weit entfernt. Oftmals halten Ärztinnen und Ärzte das Geständnis, dem Patienten ohne böse Absicht einen Schaden zugefügt zu haben, bereits für ein Eingeständnis eigener Inkompetenz. Deshalb wird meist geschwiegen. Offene Debatten sind immer noch eine Rarität.

Das Problem beim Umgang mit Fehlern ist, dass sie sofort zu persönlicher Schuld werden. Fehler sind immer unerwünschte Ereignisse. Keiner hat sie gewollt, und doch sind sie passiert. Jeder/jede Arzt/Ärztin wird mehrmals in seiner/ ihrer Berufslaufbahn davon eingeholt.

Fehlervorwürfe betreffen vornehmlich die operativen Disziplinen und in überdurchschnittlichem Masse Spitalärzte, also jene Fachrichtungen, die in Diagnostik und Therapie am stärksten in den menschlichen Körper eingreifen. Rund $60 \%$ aller gutachterlichen Bescheide in Deutschland beziehen sich auf die Fachrichtungen Chirurgie, Gynäkologie und Orthopädie. Als Beispiele werden Schwierigkeiten bei minimal invasiven Eingriffen sowie Mängel im geburtshilflichen Management genannt [16]. Allerdings geht das Robert-Koch-Institut davon aus, dass dies kein getreues Abbild der Realität ist, sondern 
eher ein Wahrnehmungseffekt. Denn sieht man von fachspezifischen Problemen ab, werden vorrangig drei Gründe für die Entstehung von medizinischen Behandlungsfehlern deutlich: organisatorische Defizite, Dokumentationsdefizite und die Behandlung von Patienten in nicht optimal geeigneten Einrichtungen [17]. Sponsel prägte in diesem Zusammenhang den Begriff der «Iatrogenie», krank durch Behandlung [18]. Er stellt sich unter anderem die Frage, ob Menschen gesünder oder länger leben, wenn sie nicht medizinisch behandelt werden.

\section{Was bedeutet Risk Management?}

Risk Management bedeutet den professionellen Umgang mit Risiken mit dem Fokus, diese zu minimieren und Chancen zu optimieren. Es ist somit eine Managementmethode, die das Ziel hat, in einer systematischen Form Fehler und ihre Folgen zu erkennen, zu analysieren und zu vermeiden. Risk Management setzt an, bevor Fehler auftreten und Schaden verursachen. Es garantiert die Sicherheit einer Institution. Sicherheit heisst aber nicht Abwesenheit von Fehlern, sondern die Fähigkeit, mit Risiken und Gefahren umgehen zu können, um so Schäden und Verluste zu verhindern und trotzdem die gesteckten Ziele zu erreichen. Risk Management ist für die Sicherung des nachhaltigen Erfolges immer wichtiger, wobei auch innerhalb eines Fehlererkennungssystems moderne Managementinformationssysteme entsprechend ausgebaut werden müssen, um eine detaillierte Riskerkennung und -bewertung $\mathrm{zu}$ ermöglichen. Vermutlich wird sich in diesem Bereich ein eindeutiger Gestaltungsbedarf ergeben, welcher grossen Einfluss auf das ärztliche Arbeitsumfeld haben wird.

So hat z.B. die US-Regierung einen Aktionsplan zur Fehlervermeidung in der Medizin veröffentlicht, welcher unter anderem die Implementierung von Fehlerbekämpfungsprogrammen in Kliniken vorsieht sowie die Etablierung eines «Centers for Quality Improvement and Patient Safety» mit einem Budget von 20 Mio. Dollar im Jahre 2000 zur Finanzierung von Forschungsprojekten oder zur Entwicklung nationaler Ziele für die Patientensicherheit [19].

Die Bewertung der Gebrauchstauglichkeit eines Qualitätssystems muss durch die oberste Verantwortlichkeitsebene erfolgen. In der Praxis ist es leider oft der Fall, dass diese Aufgabe an die Qualitätsmanagementbeauftragen delegiert wird. Das zeigt, dass vielerorts Qualität noch nicht als Chefsache angesehen wird. Wenn ein Qualitätssystem von der Geschäftsleitung als strategisches Unternehmensinstrument betrachtet wird (was in einem Spital als Selbstverständlichkeit gelten sollte), so sollte es auch die Geschäftsleitung selbst sein, welche die Beurteilung vornimmt und die Vorgaben im Qualitätssystem festlegt [6].

Es ist heute für ein modernes Sicherheitsverständnis unumgänglich, dass mehr Gewicht daraufgelegt wird, dass durch die Verbreitung des Wissens um die Entstehungsmechanismen eines Zwischenfalls die Anzahl der schwerwiegenden Ereignisse gesenkt wird. Eine diesbezügliche systematische Prophylaxe fehlt vollständig. Jeder Zwischenfall verursacht neben dem menschlichen Leid auch hohe Kosten. Vorbeugung und Verhütung sind also auf jeden Fall kostendämpfend. Aus diesem Grunde wurde bereits 1998 mit der Motion Günther eine Kommission zur Untersuchung schwerer medizinischer Zwischenfälle beantragt [20]. Leider hat diese Motion bis heute noch nichts gebracht. Um so effektiver sind dagegen die Bemühungen von privater Seite, wie z.B. der Schweizerischen Gesellschaft für Anästhesie und Reanimation (SGAR), welche im Umgang mit Fehlern eine klare Vorreiterrolle übernommen hat. Ziele der von der SGAR errichteten Stiftung zur Erhöhung der Patientensicherheit sind, möglichst viele Zwischenfälle zu erfassen und zu analysieren, diese zu kommunizieren und Aktivitäten zur Verhinderung möglicher Zwischenfälle abzuleiten. Auf der Webseite der SGAR können schweizerische Anästhesiologen unter Wahrung der Vertraulichkeit von Zwischenfällen berichten. Auf diese Weise können Informationen über seltene unfallträchtige Situationen an die gesamte Gemeinschaft der Anästhesisten weitergegeben werden, um zu verhindern, dass nicht an anderer Stelle ein ähnlicher Zwischenfall passiert.

Genauso wie in der Luftfahrt versucht das Team um Professor Scheidegger an der Universität Basel, durch das Üben und Beurteilen von Teamleistungen im OP und durch das Herausfiltern von Fehlern, welche meist aufgrund mangelhafter Kommunikation entstehen, einen hohen Sicherheitslevel zu erreichen. Die Gesamtleistung ist immer gleich dem Produkt der Einzelleistungen [21]. Wie die Luftfahrt ist auch die Medizin von entsprechenden Teamleistungen abhängig und der Teamfehler kann als Aktion bzw. Unterlassung von notwendigen Tätigkeiten definiert werden, der zu einem Abweichen vom Team- oder Organisationsziel führt [22].

Die Frage, warum es zu einem Fehler gekommen ist, und die Antwort, was getan werden kann, damit sich derselbe Fehler nicht mehr wiederholt, sollte zu einem äusserst wirksamen Lernereignis führen. Aus der Fehleranalyse können neue, bessere und sichere Prozessabläufe determiniert werden. 
Allerdings müssen die rechtlichen Fragen zwingend und vordringlich gelöst werden. Ohne garantierte Anonymität entspräche eine Fehlermeldung faktisch einer Selbstanklage. Ohne eine vorausgehende juristische Klärung kann keine neue Fehlerkultur aufgebaut werden. Entscheidend sind hierbei die Schaffung neuer gesetzlicher Grundlagen für Meldung und Untersuchung von fehlerbedingten Ereignissen oder Beinaheereignissen. Wie Kuhn in eindrucksvoller Weise feststellte, ist die systematische Fehlerursachenforschung in der Schweiz klinisch tot, bevor sie richtig begonnen hat. Es sei denn, dass der Gesetzgeber verbindlich zum voraus definiert, welche Meldungen ohne Sanktionen bleiben, d.h. dem Gutachter, dem Richter, der Gesundheitsdirektion und den Versicherern vorgelegt werden dürfen [23]. Wichtig hierbei ist auch ein breiter Konsens über einheitliche Erfassungsverfahren für Zwischenfälle mit Entwicklung klinischer und administrativer Informationssysteme unter Nutzung moderner Technologien [24].

Wo liegt aber der Cut-off zwischen Fehler ohne persönliche Schuld und nicht tolerierbarer Schlamperei? Ein Meldesystem wird letztere nicht ausrotten können und darf und will kein rechtsfreier Raum dafür sein [25]. Die Unterscheidung zwischen «alles Mögliche tun» und der «Ästhetik des Unterlassens» wird noch zu interessanten Diskussionen Anlass geben [26].

Aufgrund der Gutachteranalysen weiss man, dass der Fokus ganz klar auf die besondere Bedeutung des vertrauensvollen ärztlichen Gesprächs gelegt wird. Wenn einem eine Operation nicht so gelingt, wie dies gewünscht ist, so sollte man sich ganz besonders um diese Patienten kümmern und auch über die aufgetretenen Probleme sprechen und sie erläutern. Die meisten Verfahren vor Kommissionen und Gerichten resultieren aus Unkenntnis, Unverständnis und Misstrauen [27]. Solche Patienten bedürfen einer ganz besonderen Betreuung und gehören nicht nur durch die Assistentinnen und Assistenten visitiert. Die Ärzteschaft muss aufpassen, dass sie nicht selbst das Misstrauen in die Patienten pflanzt. Entsteht nach einer Laparotomie ein Entzündungsbauch, weil ein Bauchtuch vergessen worden war und der Chirurg nach der Revision die Kollegen und das Pflegepersonal zum Stillschweigen verdattert und unter Umständen auch noch sogenannte Dokumentationsbegradigungen vorgenommen werden, so sollte ein derartiges Vorgehen richtigerweise nicht geheim bleiben. Aus dem Behandlungsfehler, der möglicherweise als nicht schuldhaft, sondern als typische Komplikation des Eingriffs hätte gewertet werden können, wird ein Akt mit strafrechtlichen Folgen. $\mathrm{Zu}$ seinen Fehlern stehen, sie kommunizieren und nach Ursachen suchen, um ein nochmaliges Auftreten eines solchen Zwischenfalls verhindern zu können. Dies wäre das richtige Vorgehen.

Auch die Schweizerische Patientenorganisation begrüsst die Bemühungen des «incidents reporting». Auch sie unterstützen Projekte wie den momentan durchgeführten Pilotversuch der SGGG, sofern die Leistungserbringer beim Offenlegen ihrer Fehler nicht bestraft werden, sondern ihre Fehler analysieren, Verbesserungsvorschläge erarbeiten und diese umsetzen können. Nur somit kann das angestrebte hohe Niveau der Patientensicherheit erreicht werden.

\section{Von der Idee zum Erfolg}

Fasziniert von der Idee und den diversen Meldungen in den Zeitschriften wollten wir innerhalb unserer Klinik ein eigenes Projekt starten. Wir wollten nicht auf andere warten und Erfahrungen in diesem Bereich sammeln, bevor den Spitälern irgendwelche Vorgaben seitens Behörden aufdoktriniert werden. Wir entwickelten einen Fragebogen, welcher vollständig anonym ausgefüllt wird, welcher kurz und überall griffbereit ist und von jedermann problemlos ausgefüllt werden kann (einfache Sprache). Damit das Projekt zum Erfolg wurde, war vor allem die Unterstützung durch die Führung essentiell, aber auch der Einbezug der Pflege sowie die Motivation der Mitarbeiter während Fortbildungen, durch Artikel in der Hauszeitung und E-MailAnschriften.

Gemäss Gedankenmodell nach St. Galler Ansatz zum Risk Management versuchten wir, mit dem Ziel der Sicherheit als Leitidee, mit dem Fragebogen eine Bewusstseinsbildung herbeizuführen und das Gefühl der Unsicherheit zu konkretisieren [28].

Danach werden die Fragebögen alle 3 Monate bewertet und analysiert, die Risikolage identifiziert und zusammen mit der Pflegeleitung ausgewertet. Wenig später findet eine Feedbackrunde statt, wobei die Suche nach Lösungsansätzen im Plenum mit allen Mitarbeiterinnen und Mitarbeitern vorgenommen wird. Dies ist wichtig, da gerade diejenigen, welche direkt mit dem Problem konfrontiert waren, oft die besten Vorschläge haben. Somit werden Bewältigungsstrategien entwickelt, und der Kreislauf wird über die erhöhte Sicherheit wieder geschlossen.

Was uns interessiert, sind die sogenannten «Beinahezwischenfälle», welche ein enormes 
Verbesserungspotential für die Menschen, die Arbeitsstrukturen und Prozessabläufe bieten. Diese kommen häufig vor, werden kaum wahrgenommen und erst aus ihnen entwickelt sich in ihrer Gesamtheit ein gravierender Fehler. 300 Beinaheunfälle bilden die statistische Grundlage für 30 mittelschwere Vorkommnisse und diese wiederum sind die statistische Basis für einen Desasterfall (Kunstfehler) [29]. Und genau diese Beinaheunfälle gilt es zu verhindern. Die meisten Mitarbeiter/innen sind Experten auf ihrem Arbeitsgebiet, sie kennen ihren Arbeitsprozess sehr genau. Die kleinen Bagatellfehler, die im gewohnten Arbeitsablauf passieren, die jeder aus seiner Tagesarbeit kennt, sollten dann gemeldet werden, wenn man sie zuerst entdeckt oder wenn sie sogar regelmässig auftreten. Somit ist das zentrale Element eines TQM-Systems (Total Quality Management), inwieweit es gelingt, die Mitarbeiter auf die Fehlersuche in ihrem eigenen Arbeitsbereich zu schicken. Auch die Patientenbeschwerden sollte man als Chance nutzen, Schwachstellen $\mathrm{zu}$ erkennen und daraus Lösungsmöglichkeiten abzuleiten, um schliesslich wieder die Patientenzufriedenheit zu erhöhen und eine Kosten- und Qualitätsverbesserung der internen Abläufe sowie Steigerung der Mitarbeitermotivation zu erreichen.

Und welche langfristigen Ziele werden damit verfolgt? Qualitätsverbesserung der Gesundheitsversorgung, Optimierung der Patientensicherheit und Kostenreduktion.

\section{Nur wer präzis analysiert, kann richtig reagieren}

Innerhalb von 18 Monaten haben wir 72 Meldungen zusammengetragen. 22\% fielen auf den Bereich Diagnostik, 59\% auf den Bereich der Behandlung. Insgesamt waren $49 \%$ Organisationsund Kommunikationsunzulänglichkeiten, nur $5 \%$ technische Fehler, $7 \%$ betrafen die Infrastruktur sowie $37 \%$ den Bereich «human error», welcher allerdings schwierig von den anderen Untergruppierungen abzugrenzen ist.

Bei der Schweregradeinteilung ergeben sich $67 \%$ leichte, $21 \%$ mittlere und $12 \%$ schwere Fälle.

Von zentraler Bedeutung ist es, dass für die Meldenden auch ein persönlich spürbarer Nutzen im Sinne eines Fortbildungseffektes entsteht. Die Qualität des Meldeformulars spielt eine wichtige Rolle. Ein computergestütztes Meldesystem würde die Auswertung erleichtern und rationalisieren.

\section{Fehlerklassifikation}

Fehler können von unterschiedlicher Natur sein, so dass sich für den Umgang mit Fehlern recht unterschiedliche Konsequenzen ergeben können. In der Literatur werden deshalb verschiedene Klassifikationen von Fehlern vorgeschlagen, auf welche an dieser Stelle nicht speziell eingegangen wird.

Nach Reason gibt es grundsätzlich zwei Modelle für Fehlerarten: Einerseits das personenzentrierte Modell, wobei der Fokuspunkt eine Person oder eine Gruppe ist, welche sich für den Fehler verantwortlich zeichnet; andererseits das Systemmodell, fokussiert auf die Bedingungen, unter denen Personen arbeiten und auch entsprechende Verhaltensmuster entwickeln. Gemäss Reason ergeben sich folgende Schlussfolgerungen: «We can not change the human condition, but we can change the conditions under which humans work.» $[30,31]$

Der Psychologe James Reason unterscheidet zudem zwischen aktivem Versagen und latenten Gefahren [32]. Aktives Versagen entsteht bei denen, die in einem System die Leistung erbringen, dazu gehören Piloten oder eben Chirurgen. Latente Gefahren drohen aus fehlerhaften Entscheidungen der Leitung, unzureichender Ausstattung, überarbeitetem Personal, zeitlichem Druck, Kommunikationsfehlern. Latente Gefahren decken die Schwächen einer Organisation auf.

Leape unterteilt die Ursachen in krankheitsimmanente Faktoren (unbeeinflussbar), in behandlungsimmanente Nebenwirkungen/Komplikationen (z. B. Nebenwirkungen einer Tumortherapie) und in Behandlungsfehler durch:

a) organisatorische Unzulänglichkeiten;

b) technische Unzulänglichkeiten;

c) unzureichende Sorgfalt von Ärzten/Therapeuten [33].

Wie auch bei unseren Resultaten deutlich festgestellt, machte bereits Leape in mehr als $3 / 4$ aller identifizierten unerwünschten Arzneimittelwirkungen primär Systemfehler ursächlich für die Probleme verantwortlich [34]. Diese und andere Ergebnisse legen den Schluss nahe, dass Bemühungen zur Fehlervermeidung dann besonders erfolgreich sein können, wenn Veränderungen auf der Systemebene vorgenommen werden, d.h., wenn z. B. bei Ablaufprozessen Korrekturen vorgenommen werden oder bei der finanziellen, technischen, personellen Ressourcenallokation seitens der Führungsebene Massnahmen ergriffen werden. 
Der Systemanalytiker Wilpert teilt die Systemsicherheit im Gesundheitswesen in eine individuelle, systematische, lernende und kulturelle Perspektive ein. Dabei ist die individuelle Perspektive jene, die in der Medizin am häufigsten beachtet wird, und zwar von den Betroffenen (Arzt, Patient, Pflege) genauso wie von den Medien. Wilpert meint, dass wahrscheinlich das traditionelle Bild des Arztes als Heiler und die Kunst des Heilens über lange Zeit einen rationalen Zugang zur Analyse dieses Systems behindert hat. Die Frage bei entstandenen Fehlern, in der Psychologie definiert als Nichterreichen eines Handlungszieles, sollte eben nicht sein «Wie konntest du das tun?», sondern vielmehr «Wie konnte es dazu kommen?» [35]. Gemäss Wilpert ist die Ebene des Systemdenkens in der Medizin noch weitgehend unbedeutend. Damit meint er, dass eben nicht nur das Individuum Fehler machen kann, sondern dass Fehler entstehen oder latent vorhanden sind, die eigentlich dem Gesamtsystem angelastet werden müssen.

\section{Fehlerursachen}

Fehlhandlungen in komplexen Systemen wie in einer Klinik dürfen nicht isoliert betrachtet werden. Sie müssen immer im Kontext aller beteiligten Einflussfaktoren gesehen werden. Hierzu gehören die beteiligten Personen, die technischen Bedingungen, die organisationalen Elemente wie Arbeitszeiten oder Dienstanweisungen sowie gesellschaftlich verankerte Einflussfaktoren wie z.B. finanzielle Rahmenbedingungen der Krankenkassen.

Gemäss Wehner lernt man ein System erst durch Fehler besonders gut kennen. Wer die Fehler seines Systems nicht kennt, kann nicht wirklich adäquat damit umgehen. Erst die Betrachtung von Fehlern ermöglicht Einblicke in ein System, welche im Routinebetrieb nie möglich wären [36].

Die internationalen Analysen stimmen darin überein, dass medizinische Fehler vorrangig aus Systemfehlern, d.h. aus Organisationsmängeln resultieren. Individuelle Versäumnisse sind dagegen nur nachrangig von Bedeutung.

\section{Belastungsfaktor Arbeitszeit}

Der zunehmende Druck der Öffentlichkeit stärkt zunehmend das Bewusstsein für ein effizientes Fehlermanagement [37]. Meistens werden jedoch die technischen Aspekte von Fehlern am Arbeitsplatz erforscht. Menschliche Fehler werden erwähnt, ohne den Gründen genau nachzugehen. Gründe werden, wenn überhaupt, eher dem fehlenden Training und dem Management zugeschrieben als der fehlenden Leistungsfähigkeit aufgrund von Ermüdung, obschon die langen Arbeitszeiten bei Medizinern als Quelle von medizinischen Fehlleistungen jährlich hohe Kosten verursachen [38].

Behandlungsfehler können oft nicht mit besserer individueller Ausbildung im jeweiligen Beruf verhindert werden. Entscheidend für das Entstehen schlimmerer Fehler sind heute vielmehr das Praxisumfeld und die mit den gesteigerten Anforderungen verbundenen Stressfaktoren. Die Zwischenfälle, welche sich in der Medizin in neuester Zeit ereignen, sind gemäss Meister kein Zufall [39]. Sie seien Alarmzeichen für das, was sich im Gesundheitswesen in der Schweiz abspiele. Vom Medizinpersonal werde immer mehr gefordert, ohne dass die entsprechenden Ressourcen (Zeit!) zur Verfügung gestellt werden. Interessanterweise ist vielen Diskussionen um medizinische Fehlleistungen nicht zu entnehmen, dass die Arbeitsbelastung durch Schicht- und Nachtdienst, unzureichende und oft gestörte Pausen sowie langdauernde Arbeitszeit auch als Fehlerquelle gesehen wird [40]. Wenn man das Bewusstsein für ein effizientes Fehlermanagement stärken und den Ursachen auf den Grund gehen will, so muss man in der grossen Gruppe der oben beschriebenen «human errors» und Organisationsmängel auch die Problematik der zu langen Arbeitszeiten bei Medizinern betrachten. Es ist zu befürchten, dass wir durch den permanenten Zeitdruck und durch ungenügende personelle Dotierungen den Zenit der maximalen Sicherheit bereits überschritten haben. Diesbezüglich wurde eine interessante Studie von Frau Riedo vom Psychologischen Institut der Universität Zürich mit der Fragestellung veröffentlicht, ob zunehmende medizinische Fehlleistungen mit den hohen Arbeitszeiten von Ärztinnen und Ärzten einen Zusammenhang haben [41]. Werden medizinische Fehlleistungen durch die Arbeitssituation von Ärzten begünstigt? Dreimal dürfen Sie raten!

Sie konnte in ihrer Arbeit Ermüdung als Quelle von Fehlleistungen ganz klar belegen. Da Ermüdung als Zustand nicht direkt erkennbar ist, ist es notwendig, wie das in der Luftfahrt gehandhabt wird, den Faktor Ermüdung mit angemessenen Arbeitszeiten als Fehlerquelle vorweg zu eliminieren. Dadurch könnte neben der Verminderung der Fehlleistungen auch die Produktivität verbessert werden, und die Qualität für unsere Patientinnen und Patienten würde um einiges reicher.

Ackerstedt konnte zeigen, dass normale Schichtarbeit bei Taxifahrern zu zunehmender 
Unfallanfälligkeit führte [42]. Pikettdienst hat eine ähnliche Wirkung auf die physiologischen Parameter und führt ebenfalls zu Schlafstörungen, weil während des Pikettdienstes das Aktivationsniveau erhöht ist. Dieser Zustand betrifft offenbar fast alle Individuen und ist verbunden mit Beeinträchtigung der Leistungsfähigkeit in einem Ausmass, in dem eine erhöhte Unfallrate erklärt werden kann. Schichtarbeit führt aufgrund unregelmässigen Schlafes und zu kurzen Ruhezeiten zu Ermüdung. Die zirkadianen Rhythmen sind gestört, körperliche Symptome wie Schlafstörungen nehmen zu, die kognitiven Leistungen wie z.B. die Denkleistung sind reduziert und die zentralen und vegetativen Funktionen eingeschränkt. Diese Symptome bewirken eine Reduktion der Leistungsfähigkeit, was Fehlleistungen begünstigt und die Produktivität verringert [35].

\section{Zusatzfaktor Selbstüberschätzung}

Arbeiten, wenn man erschöpft ist, wird in der Medizin als professionell angesehen. Sexton befragte 3000 Piloten sowie über 1000 Ärzte in bezug auf die Selbsteinschätzung in bestimmten Situationen. Interessant war die Aussage: «Auch wenn ich übermüdet bin, bin ich in der Lage, in Notfallsituationen effektiv zu handeln», welche nur 26\% der Piloten, aber 70\% der Chefärzte mit «ja» beantworteten [43].

Unabhängig von der Qualifikation führt Erschöpfung durch hohe Arbeitszeiten jedoch zu medizinischen Fehlleistungen. Eine 80-StundenWoche, inkl. 40 Std. unbezahlter Arbeit und keinen Tag frei, wird in der Privatwirtschaft nicht akzeptiert, in der Medizin hingegen schon [44]. Helmreichs Vergleich mit der Luftfahrt ist bezeichnend: In der Luftfahrt wird trainiert, Erschöpfungs- und Stresssymptome wahrzunehmen. Dadurch wurden Fortschritte gemacht, die Fehlerquelle zu eruieren, während in der Medizin immer noch versucht wird, Fehler zu verdecken [45]. Oder anders gesagt: In der Fliegerei ist es schwer, Fehler zu machen, und leicht, sie zu entdecken. In der Medizin ist es leicht, Fehler zu machen, und schwer, sie zu entdecken [46]. Studien haben gezeigt, dass Mediziner sich gerne unantastbar geben, sie stehen über ihren Fehlern! [43] Ebenso spielen sie den Effekt von Erschöpfung und Stress herunter. Die Ablehnung von Stress und die daraus resultierenden Konsequenzen helfen, sich dem medizinischen System anzupassen. Aber das Erkennen und Wahrnehmen von Stressoren und deren Konsequenzen würde mithelfen, Fehler zu reduzieren [35].

Das Problem ist nur, während die Arbeitszeiten des Personals der Luftfahrt geregelt sind und
Missbrauch strafrechtlich verfolgt wird, werden in der Medizin sehr hohe Arbeitszeiten sogar dort geduldet, wo eindeutige gesetzliche Vorschriften entgegenstehen [47].

\section{Belastungsfaktor Psyche}

Jede Organisation ist eine Schnittstelle von Institutions-/Gruppen-/Familien- und Psychodynamik. Das einzelne Individuum befindet sich ständig im Austausch mit seiner Familie, mit der Gruppe als sozialer Haut (z.B. am Arbeitsplatz) sowie der Organisation als Institutionelles a priori [48].

\section{Belastungsfaktor Umgebungseinflüsse}

Lärm, extreme Helligkeit oder Wärme führen ebenso zu erschwerten Arbeitsbedingungen mit erhöhter Fehleranfälligkeit. Gerade im Operationssaal sollte es nicht zu lärmig sein und nur das Nötigste geredet werden. Auch lange OP-Zeiten können zu Übermüdung durch Schwitzen, langes Stehen und Anstrengung führen.

\section{Belastungsfaktor Messinstrumente}

Geräte können Fehlfunktionen aufweisen. Auch der Mensch als Beurteiler kann das Gerät fehlinterpretieren. Diesbezüglich ist in der modernen Medizin der Einsatz von computerunterstützenden Systemen wünschenswert und unerlässlich. Hierbei spielt die Softwaretechnik nicht nur im Bereich der medizintechnischen Geräte, sondern auch in der medizinischen Informationsverarbeitung eine wichtige Rolle. Es sei besonders das klinische Informationssystem angesprochen, das ein Kommunikations- und Beratungssystem darstellt und seinerseits einen Beitrag zur Fehlervermeidung im Bereich der Dokumentation und Kommunikation leisten soll (erhebliche Arbeitsentlastung und fehlerfreie Datenübermittlung!).

\section{Probleme}

Damit sei nochmals ausdrücklich auf die Wichtigkeit der Gesprächskultur hingewiesen. Die Kommunikation ist zentral. Wir alle wissen, dass das Gesagte nicht immer gehört wird, das Gehörte nicht immer verstanden wird und das Verstandene nicht einem stummen Einverständnis gleichzusetzen ist.

\section{Kulturwandel braucht Zeit}

- Zeitaufwand des Projektes. Das Entwickeln des Fragebogens, das Sammeln, Bewerten und Analysieren der Daten sowie Besprechen im Plenum braucht Zeit und Geduld. 
- Die Motivation der Mitarbeiter und Mitarbeiterinnen spielt eine grosse Rolle. Sobald es an Feedback fehlt, fällt die Motivation deutlich zurück.

- Schwieriger Kulturwandel.

- Oftmals harzige Kommunikation.

- Das Problem der Anonymität kann durch ein computerbasiertes System vollständig gelöst werden.

- Gefahr des Underreporting, d.h., banale Ereignisse können untergehen.

Fit für den Kulturwandel sind wir nur dann, wenn wir bereit sind, alles immer in Frage zu stellen und Veränderungen Wirklichkeit werden zu lassen! Damit uns dies vollumfänglich gelingen mag, brauchen wir auch die Entwicklung der entsprechenden gesetzlichen Grundlagen für sanktionsgeschützte Meldeverfahren. Von einer Fehlerkultur sind wir noch weit entfernt.

Wichtig ist es, den Kurs weg von einer oberflächlichen reaktiven Kultur der Schuldzuweisung zu finden, hin zu einer systemanalytischen proaktiven Sicherheitskultur, die auch als präventive Fehlerkultur bezeichnet werden kann [49]. Dieser Wandel wird weder einfach noch schnell zu vollziehen sein. Und denken wir daran, dass es wahrscheinlich in der Natur des Menschen liegt, Fehler zu machen, aber ebenso auch Lösungen zu entwickeln und Alternativen zu finden, um sich den Herausforderungen der Zukunft stellen zu können [3].

Grundfähigkeiten wie Führung, Beobachtung, Kommunikation und gegenseitige Überprüfung sollten gelehrt werden. Die Fehlerkultur zielt nicht darauf ab, einzelne Fehler zu verhindern, sondern vielmehr ein System zu fördern, welches mit Fehlern umgehen kann. Auch Reason beschreibt vier wichtige Komponenten einer effektiven Sicherheitskultur: ein adäquates Meldesystem, angemessene Reaktionen auf die erhaltenen Meldungen, Flexibilität und schliesslich die Fähigkeit, aus Erfahrungen lernen zu können.

\section{Lösungsansätze}

- Es benötigt einen Projektleiter als verantwortlichen Ansprechpartner, welcher die Kontrolle übernimmt und für eine tolerante Projektkultur besorgt sein sollte.

- Die Schnittstellen zwischen Mensch, Technik und Spital als Unternehmen sollte man verstehen, insbesondere die darin verwobenen dynamischen Ebenen als Systemfehler- quellen erkennen (Institutions-/Gruppen-/ Familien-/Psychodynamik).

- Menschliches Handeln und die damit verbundenen Fehlbarkeiten anerkennen (psychologische Aspekte).

- Teamförderung Pflegende/Ärzte.

- Regelmässiges Feedback (Mitarbeiter als Mitdenker anerkennen).

- Computergestütztes Meldesystem.

- Vorbehaltloses Kommunizieren von Fehlern und Missgeschicken macht deren Behebung möglich. Versuchen, von den Fehlern anderer zu lernen, darauf bauen, eine Vertrauensatmosphäre bilden. Versuchen, aus dem eigenen Elfenbeinturm herauszukommen, und das sogenannte «not invented here»-Syndrom bekämpfen, denn schliesslich leben wir nicht lange genug, um alle Fehler selbst zu machen.

\section{Ausblick}

Innerhalb unseres Spitals versuchen wir, das Zwischenfallmeldesystem auf die gesamte Klinik auszubauen. Der Fragebogen muss dabei je nach Klinik angepasst werden, wobei ein gemeinsamer Konsens angestrebt werden sollte.

Wünschenswert wäre ein nationales Institut mit der Aufgabe zur Untersuchung schwerer medizinischer Zwischenfälle. Die professionelle Kommunikation der Lehren aus Zwischenfällen wäre eine wichtige Aufgabe dieses Institutes. Die breite Kommunikation dieser Lehren aus Fehlern ist die beste Prophylaxe gegen künftige Fehler. Darüber hinaus wäre eine Koordination über die Grenzen hinaus wünschenswert.

Qualitätssicherungssysteme, insbesondere Critical-Incidents-Reporting-Systeme, müssen aber nicht unbedingt nur im stationären Bereich durchgeführt, sondern könnten auch im Rahmen der Grundversorgung verankert werden [50].

Wie auch in den Spitälern sind die Praxen als organisatorische Einheiten gefordert, für sich ein Programm zur laufenden Qualitätsverbesserung zu implementieren [51].

Die Zeiten für uns Ärzte und Ärztinnen werden nicht besser werden, im Gegenteil, der Wettbewerb untereinander wird zunehmen. Das individuelle und öffentliche Eingestehen von Fehlern ist schwierig und wird mit Sicherheit nicht einfacher werden. Es kommen Probleme hinzu wie Informationsverluste angesichts des Einhaltens von Arbeitszeitgesetzen, die mangelnde Finanzmasse zur Begleichung von Über- 
stunden, der Motivationsverlust der ärztlichen Mitarbeiter/innen angesichts düsterer Zukunftsprognosen. Andererseits werden die Ansprüche der Patienten an die so teure Krankenhausmedizin weiterwachsen.

Wir brauchen die Möglichkeit, anonym an eine loyale, aber unabhängige Stelle zu berichten. Dort können Fehlerberichte, die für alle gelten, systematisch gesammelt und durchforstet werden. Es könnten Szenarien entwickelt werden, welche einer Wiederholung entgegengestellt werden müssen [52].

\section{Fit für den Kulturwandel?}

Ein Fehlermeldesystem kann zu einer laufenden Qualitätsoptimierung führen. Voraussetzungen sind aber ein Klima des Vertrauens. Die Meldung von Fehlern entlastet die Kolleginnen und Kollegen auch von Schuldgefühlen und führt zu einem Lerneffekt für alle Beteiligten. Sie werden dazu ermuntert, ihr Handeln immer wieder selbstkritisch zu reflektieren. Wichtig ist vor allem auch das gute Beispiel der Leitung, welche auch über eigene Fehler berichten sollte. Ein konstruktiver Umgang mit Fehlern kann sich nur im gegenseitigen Respekt unter ehrlicher kollegialer Offenheit und Bereitschaft von allen Beteiligten zur Selbstbescheidenheit entwickeln.

Literatur

1 Neue Zürcher Zeitung 12/99.

2 Robert-Koch-Institut, Statistisches Bundesamt, Gesundheitsberichterstattung Heft 04/01.

3 Cohn LT, Corrigan JM, Donaldson MS. To Err is Human - Building a Safer Health System. Washington, DC: National Academy Press; 1999.

4 Ollenschläger G. FMH-Symposium: Risiken, Fehler und Patientensicherheit. Bern, 8. Januar 2001.

5 Möndmann HG. Achtung Arzt! Erstklassige Medizin - drittklassige Behandlung. München: Dröhmer; 2000.

6 Krankenhaus-Risk-Management. www. asstech.com/de.

7 www.aezq.de.

8 DBV Winterthur. Ergebnis Statistik 2000.

9 Ollenschläger G. Die Problematik der Fehler und der Patientensicherheit in Deutschland. FMH-Symposium: Risiken, Fehler und Patientensicherheit. Bern, 8. Januar 2001.

10 Rall M, Manser T, Buggenberger H, Gaba D, Unertl K. Patientensicherheit und Fehler in der Medizin - Entstehung, Prävention und Analyse von Zwischenfällen. Anästhesiol Intensivmed Notfallmed Schmerzther 2001;36:321-30.
11 Vincent C, Neil G, Woloshynowych M. Adverse events in British hospitals: preliminary retrospective record review. BMJ 2001;322:517-9.

12 Scheidegger D. Aussage anlässlich Vortrag «Risk Management». Zürich, 2002.

13 Haller U. Fortbildungsseminar Projektmanagement SGGG. Zürich, November 2002.

14 Kehrer B. Fortbildungsveranstaltung Risk Management. Luzern, 2002.

15 Weiss P. Praktische Qualitätsarbeit in Krankenhäusern. ISO 9001: 2000. Total Quality Management. Wien, New York: Springer Verlag; 2000.

16 Flintrop J. Ärztekammer Westfalen Lippe: mehr misstrauische Patienten. Dtsch Ärztebl 2002; 99(27):1572.

17 Robert-Koch-Institut. Medizinische Behandlungsfehler. Gesundheitsberichterstattung des Bundes. Statistisches Bundesamt 2001(04):3-12.

18 Sponsel R, Gesellschaft für allgemeine und integrative Psychotherapie. Deutschland. Fehler, Behandlungsfehler, Kunstfehler - ein kritischer Beitrag zur Epidemiologie des Gesundheitssystems, das selbst ein wichtiger Faktor für Krankheit und Tod ist. 07/02

19 QUIC-Quality Interagency Coordination Taskforce. Doing what counts for patient's safety. Federal actions to reduce medical errors and their impact. Report to the President.

20 Motion Günther, 98.30.25, Auszug aus dem Nationalrat.

21 Nolan TW. System changes to improve patient safety. BMJ 2000;320:771-3.

22 Park K. Human Error. In: Salvendy G. Handbook of Human Factors and Ergonomics. New York: Wiley; 2001. p. 150-73.

23 Kuhn HP. Risiken, Fehler - juristische Aspekte. FMH-Symposium: Risiken, Fehler und Patientensicherheit. Bern, 8. Januar 2001.

24 Bates DW. Using information technology to reduce rates of medication errors in hospitals. BMJ 2000;320:788-91.

25 Kissling B. SGAM-Mitteilungen. PrimaryCare 2002;2:233-6.

26 SGAM-Kongress. Ästhetik des Unterlassens. Interlaken, 1999.

27 Eckernkampp A. Risk Management im Krankenhaus. Universität Greifswald, Berlin.

28 Fahrni F. St. Galler Ansatz zum Risk Management. Institut für Technologiemanagement, Universität St. Gallen.

29 von Eiff W. Heinrichs Gesetz. CKM Krankenhausmanagement. Münster, Januar 2001.

30 Reason JT. Human error: models and management. BMJ 2000;320:768-70.

31 Reason JT. Human Error. New York: Cambridge University Press, 1990.

32 Reason JT. Human Error. Cambridge, UK: Cambridge University Press; 1990.

33 Leape LL. The Preventability of Medical Injury. In: Bogner MS (ed.). Human Error in Medicine. Hillsdale NJ: Erlbaum Publications; 1994. p. 13-25. 
34 Leape LL,. Bates DW, Cullan DJ. Systems analysis for adversed drug events. JAMA 1995;274(1): 35-43.

35 Wilpert B. Sicht eines Systemanalytikers. Kongressbericht. Technische Universität Berlin, April 2001.

36 Wehner T. Sicherheit als Fehlerfreundlichkeit. Opladen: Westdeutscher Verlag; 1992.

37 Wolff AM, Bourke J. Reducing medical errors a practical guide. Med J Aust 2000;173:247-51.

38 Dinges D. An overview of sleepiness and accidents. J Sleep Res 1995;4:4-14.

39 Meister B. Medienorientierung «Mehr Sicherheit für die Patienten». Januar 2001. www.sgar.ssar.ch/ Qualität/Pat-Sich.htm.

40 Meuli U. Arbeits- und Lebenssituation der Zürcher Assistenz- und Oberärzte. Unveröffentlichte Studie im Auftrag des Verbandes Schweizerischer Assistenz- und Oberärzte, VSAO Sektion Zürich. Zürich: Soziologisches Institut; 1998.

41 Riedo V. Medizinische Fehlleistungen: Der Einfluss hoher Arbeitszeiten auf die Leistungsfähigkeit von Ärztinnen und Ärzten. Psychologisches Institut der Universität Zürich, Abteilung Sozialpsychologie 2, Juli 2001.

42 Ackerstedt P. Work hours, sleepiness and the underline mechanisms. J Sleep Res 1995;4:15-22.

43 Sexton JB, Thomas E, Helmreich RL. Error, stress and teamwork in medicine and aviation: cross section surveys. BMJ 2000;320:745-9.

44 Olson LG, Ambrogetti A. Working harder, working dangerously? Fatigue and performance in hospitals. Med J Aust 1998;168:614-6.
45 Helmreich RL. On error management: lessons from aviation. BMJ 2000;320:781-5.

46 Kehrer B. Errare humanum est. Von der Schuldzur Fehlerkultur. Vortrag anlässlich Qualitätsmanagementtagung Luzern, 2001.

47 Reck RM. Zitat. Juni 2001 aus [41].

48 Eck CD. Mit Widersprüchen umgehen. Institut für angewandte Psychologie, IAP Zürich. Vortrag anlässlich Total-Quality-Management-Tagung in Luzern, September 2001.

49 Rall M, Manser T, Buggenberger H, Gaba D, Unertl K. Patientensicherheit und Fehler in der Medizin - Entstehung, Prävention und Analyse von Zwischenfällen. Anästhesiol Intensivmed Notfallmed Schmerzther 2001;36:321-30.

50 Huber F. Critical incidents reporting in der Grundversorgung. Schweiz Ärztezeitung 2002; 83(28):1517-21.

51 Geboers H, Grohl R, van den Bosch W, van den Hoogen $\mathrm{H}$, Mokink H, van Montford P, et al. A model for continuous quality improvement in small scale practices. Qual Healthcare 1999; 8:43-8.

52 Paschen U. Menschliches Versagen? Bemerkungen zum Umgang mit unerwünschten Ereignissen. Medizinische Qualitätssicherung, Uni Hamburg, $08 / 01$ 